\title{
UN DUEL ENTRE DEUX ROMANTIQUES HERZEN ET HERWEGH
}

\author{
PAR \\ ULRICH SCHMID \\ Université de Saint-Gall
}

L'œuvre majeure d'Alexandre Herzen Passé et méditations (Byloe $i$ dumy) naît d'une double crise : en 1848, les révolutions européennes échouent, malgré les espoirs que Herzen avait placés en elles, alors que le mariage de Herzen se voit peu après ébranlé en ses fondations. La série de coups du sort ne prend pourtant pas fin avec ces événements. En novembre 1851, sa mère et son fils se noient lors d'un accident de bateau au cours d'une traversée à destination de Nice. Début mai 1852, Natalie meurt en couches, le nouveauné n'y survivra pas. La catastrophe privée et publique s'unissent en laissant apparaître un vide de sens qui demande à être comblé.

En 1852, Herzen entreprend l'écriture de son autobiographie Passé et méditations, qui l'occupera jusqu'en 1870 , date de sa mort ${ }^{1}$. Le drame conjugal qui se déroule entre 1849 et 1852 y joue un rôle particulier ${ }^{2}$. Non moins expérimenté dans les affaires d'amour ${ }^{3}$, le poète de la révolution Georg Herwegh, marié, avait entamé une liaison avec la femme de Herzen, Natalie. En novembre 1849, Natalie avorte à Genève. L'enfant était probablement de Herwegh. Herzen ne sut jamais rien de cet avortement ${ }^{4}$.

1. Ulrich Schmid, Ichentwürfe : Russische Autobiographien zwischen Avvakum und Gercen, Zürich, 2003, p. 328.

2. Simone Rist, «L'affaire Herwegh », Revue des études slaves, t. LXXVIII, 2007, fasc. 23, p. 229-242.

3. Il faut rappeler ici avant tout sa passion des années 1844 à 1845 pour Marie d'Agoult, qui avait elle-même vécu en ménage avec Franz Liszt et était devenue plus tard la belle-mère de Richard Wagner. Cf. Au printemps des dieux : correspondance inédite de la comtesse Marie d'Agoult et du poète Georg Herwegh, Paris, 1929.

4. Georg Herwegh, Briefe 1849-1875 = Werke und Briefe, t. 6, éd. Ingrid et Heinz Pepperle, Bielefeld, 2010, p. 129, 132. Cité par la suite : Lettre suivi du numéro. 
Dans l'immédiat, les deux rivaux entretiennent une amitié. Herzen avait fait la connaissance de Herwegh en 1848 à Paris, où le poète allemand et Ivan Turgenev occupaient la même maison. Leur proximité politique et idéologique les réunit bientôt sous la bannière d'un combat commun. Herwegh écrit ainsi à sa femme :

Mais tu sais combien $\mathrm{j}$ 'aime Herzen et combien nous apparaissons au cœur de cette confusio rerum comme les seuls hommes qui se comprennent encore ; c'est pourquoi je conserve cette dernière amitié comme un trésor $[\ldots]^{5}$.

Encore dans une lettre à sa femme Emma datée du 15 septembre 1849, au moment où l'affaire avec Natalie bat son plein, Herwegh rêve d'un ménage à quatre, dans lequel amitié et amour seraient reliés parallèlement et symétriquement dans une sorte de carré de sympathie idéal. Les femmes et les hommes seraient amis, et les relations entre sexes seraient possibles dans et hors du mariage :

Mes sens et mon aspiration sont tout entier dirigés vers la manière dont nous pouvons avec Herzen nous établir en quelque lieu ${ }^{6}$.

Étrangement, cette vision utopique s'est maintenue jusqu'en juin 1850, au moment même où l'amitié entre Herzen et Herwegh - du côté de Herzen tout du moins - s'était déjà transformée en haine ${ }^{7}$.

L'amour et l'engagement politique marchaient alors main dans la main. Son esprit démocrate, Herwegh le déduisait également de l'amitié et de la capacité à aimer du poète. Dans son article le Poète et l'État (1839), Herwegh explique :

Plus la méfiance des gouvernements envers la littérature était grande, plus cette dernière se renforçait. La littérature est devenue désormais un second pouvoir dans l'État, et le gouvernement peut avec sa défiance librement invoquer Platon, dont la sentence est d'autant plus vraie qu'elle s'appliquerait en premier lieu à lui-même. Lorsqu'une fois j'ai dit quelque part: tout poète authentique est en fait un démocrate, alors je dois ici reprendre, à quelque exception près, cette expression comme ce concept. André Chénier était assurément un démocrate, et la République l'a pourtant envoyé à l'échafaud. Chaque poète se trouve en opposition avec l'État, même avec le meilleur ${ }^{8}$.

Ce texte, Herzen aurait pu aussi l'écrire. L'engagement politique de Herwegh est resté pour l'émigré russe le but suprême de sa propre activité de publiciste. Largement inconnu en Europe, Herzen a fait intervenir la notoriété d'Herwegh, afin de doter ses propres écrits d'une sorte de label de qualité,

5. Lettre 11.

6. Lettre 16.

7. Lettre 89 .

8. Georg Herwegh, Frühe Publizistik 1837-1841, Glashütten im Taunus, 1971, p. 35-37, ici p. 36. 
Herwegh ayant été gratifié par Heinrich Heine en 1844 du titre de noblesse d' « d'alouette de fer », s'élevant « avec allégresse vers la lumière sacrée du soleil ».

L'étoile d'Herwegh devait pourtant pâlir après l'échec de la révolution de 1848. À la suite de la révolution parisienne de Février et des événements de Mars en Allemagne, Herwegh marche à la tête d'une légion sur Strasbourg en direction de Baden. Cette légion voulait s'élancer au secours des insurgés de Friedrich Hecker. Des escarmouches à Dossenbach conduisent Herwegh et ses troupes à une défaite honteuse. Parce qu'il s'était caché prétendument sous le revêtement en cuir d'un carrosse, il perdra toute crédibilité au sein de la communauté révolutionnaire.

Pour Herzen, la question du courage personnel de Herwegh ou de sa liberté n'est pas de première importance. L'échec de la révolution de 1848 lui apparaît alors à l'échelle historico-philosophique'. C'est pourquoi Herzen n'interprète pas l'aventure de Herwegh à Dossenbach comme une cause, mais comme le symptôme d'un événement historique. Herzen publie dans le Deutscher Monatsschrift de décembre 1850 un essai sur l'échec de la révolution, vraisemblablement traduit en allemand par Herwegh ${ }^{10}$. En outre, Herzen écrit un article sur ce sujet dans la Voix du peuple de Proudhon, qu'il dédie à Herwegh $^{11}$. Dans son livre De l'autre rive (S togo berega), qui parait d'abord en traduction allemande à Hambourg sous une indication de lieu déguisée (en vérité il fut édité à Zürich), apparaît le même texte, cette fois sous le titre de « Pour Georg Herwegh ». Herzen s'adresse avec un ton cordial au " cher Herwegh » et constate que "partis de deux extrêmes opposés, nous nous rencontrons au même point »: «Je suis venu comme étranger, en Europe ; Vous, vous vous êtes fait étranger. » Herzen achève sa longue lettre ouverte en annonçant que, si le combat révolutionnaire en Europe échoue, il émigrera aux États-Unis. Ordre était intimé à Herwegh : "Sans doute m'y rejoindrez-vous ?..... Actuellement on reçoit rarement pareilles dédicaces, on ne reçoit du reste pas souvent de dédicaces des barbares ${ }^{12}$.» Le texte indique Montreux 25 août 1849. Herzen n'est à ce moment-là pas encore au courant de l'adultère, ce qu'il reprochera plus tard à Herwegh.

9. Ulrich Schmid, «The Family Drama as an Interpretive Pattern in Aleksandr Gercen's Byloe i dumy », Russian literature, t. LXI, 2007, fasc. I-II, p. 67-102.

10. Ingrid Pepperle, «Georg Herwegh und Alexander Herzen. Unbekannte Briefe zum Bruch ihrer Beziehungen von Richard Wagner, François Wille und Georg Herwegh », in Begegnung und Zeiten: Festschrift für Helmut Richter zum 65. Geburtstag, éd. Regina Fasold, Christine Giel, Volker Giel, Michael Masanetz, Michael Thormann, Leipzig, 1999, p. 141-153, ici p. 141.

11. Judith E. Zimmerman, «Herzen, Proudhon and la Voix du peuple : a reconsideration », Russian history, t. 11, 1984, p. 422-450.

12. [Alexander Herzen,] Vom anderen Ufer : aus dem Russischen Manuskript, Hamburg [i.e. Zürich], 1850, 141 f., 180 ; Alexandre Herzen, De l'autre rive, Genève, Slatkine, 1980, p. 251. 
L'amitié entre les deux écrivains ne représente pas simplement une affaire sociale, mais devient aussi un fait littéraire. On se jure fidélité et on met en scène de cette manière le récit romantique d'une amitié, garantie d'une intensité de sentiment maximale.

L'adultère possède également une dimension romantique prononcée. De manière piquante, le roman entre Herwegh et Natalie aura trouvé son dénouement à l'heure russe. Herwegh lit en effet avec elle dans l'original Puškin, Gogol' et Lermontov. Le 15 septembre 1849 (au point culminant de leur affaire extra-conjugale), il écrit fièrement à sa femme :

Je passe la moitié de mes jours à l'apprentissage du russe, et cela en constitue la plus belle moitié. J'y ai trouvé une nouvelle source de poésie qui me procure un grand rafraîchissement; actuellement, hors de la poésie et de l'amour rien ne subsiste. Natalie possède une compréhension importante et fine, et sait au moins appréhender ses poètes avec lyrisme. Ces heures me procurent un plaisir infini et il ne manque plus que tu les partages avec nous ${ }^{13}$.

La seconde partie ne doit pas être comprise comme un aveu de cynisme. Herwegh croyait sincèrement dans le fait qu'il pourrait unir - comme dans un roman d'amour utopique - sa passion pour Natalie et son amour pour Emma.

Herwegh met en scène sa romance avec Natalie Herzen d'après le modèle de la Nouvelle Héloïse de Rousseau. Dans ce roman, le mariage entre Julie et Monsieur de Wolmar est largement ouvert à l'ancien amant Saint-Preux. Au moyen de ce récit, une légitimation valable était trouvée pour l'intrusion d'Herwegh dans le mariage des Herzen. La localisation romantique de celui-ci sur les rives du lac Léman jouait aussi un rôle important. Le roman de Rousseau se déroule à Clarens, alors que l'histoire d'amour entre Herwegh et Natalie Herzen commence en août 1849 avec une ascension commune de la Dent de Jaman, montagne située au-dessus de Clarens. Natalie utilisera dorénavant sans cesse dans ses lettres le signe du destin $\Lambda$, qui signifie ici déclaration d'amour échangée au sommet de la montagne ${ }^{14}$.

Pour Herwegh il n'y avait, par rapport à la trame de cette folie littéraire, aucune contradiction dans le fait qu'il parte encore le même mois avec Herzen pour une excursion en montagne sur le Monte Rosa. À l'origine de cette marche se trouve également un texte littéraire : le poème dramatique de Byron Manfred (1817), dans lequel le protagoniste entretient une conversation à deux avec la nature au sommet de la Jungfrau. Cette excursion était

13. Lettre 16, Victor Fleury, le Poète Georges Herwegh, Paris, 1911, p. 156. Fleury ne mentionne l'affaire Herzen que comme un «drame intime » et marque dans une note de bas de page : «Le moment n'est pas encore venu de révéler au public toutes les circonstances de ce drame qui n'intéresse d'ailleurs en aucune manière l'œuvre que j'étudie. » (p. 155).

14. E. H. Carr, the Romantic Exiles : a nineteenth-century portrait gallery, London, 1933, 59. 
pour Herzen une répétition de son serment de jeunesse sur le mont des Moineaux (Vorob'ëvy gory) de Moscou, lorsqu'il voulait avec Ogarev consacrer sa vie à la chose révolutionnaire. Lorsque Herwegh et Herzen reviennent de leur excursion en montagne, ils sont - comme Natalie l'écrit dans une lettre à Emma Herwegh - «bronzés, heureux et satisfaits comme des enfants ${ }^{15} »$.

Leurs contemporains également considéraient aussi l'adultère comme un modèle romantique. Pavel Annenkov caractérisait Herwegh comme une sorte de "Lohengrin », apparaissant à Natalie Herzen depuis " des hauteurs fabuleuses $»$ :

Ce personnage étrange recelait en lui des trésors d'égoïsme, des penchants épicuriens et la nécessité d'éprouver et de libérer ses passions à n'importe quel prix, sans se préoccuper des sacrifices qui tombaient sous le couperet de son égoïsme fou ${ }^{16}$.

Herzen décrit finalement lui-même son couple au moyen d'une interprétation littéraire. Le Bildungsroman constitue ici un modèle important, en particulier les Affinités électives de Goethe ${ }^{17}$. Herzen mentionne déjà le roman dans une lettre du 26 février 1841 et en identifie le noyau dramatique comme la collision entre «le formalisme du mariage» et «la sympathie naturelle ${ }^{18}$. Herzen essayait aussi d'extraire son propre couple du domaine des conventions sociales afin de lui conférer la dignité d'une unité métaphysique plus élevée.

Tant Herwegh que Herzen interprétaient la situation avec des morceaux de sens tirés des romans de George Sand ${ }^{19}$. Il s'agit justement de la réalité que George Sand recrée sans cesse au moyen d'une constellation triangulaire, et dont les romans sont pris comme modèle pour interpréter la rivalité des deux auteurs dans leur quête des faveurs érotiques de Natalie Herzen. Sand transforme le même thème dans des variations toujours nouvelles qui pourront être à chaque fois actualisées aux différents stades de la relation entre Herzen et Herwegh.

Le double roman Consuelo et la Comtesse de Rudolstadt présente une conception harmonieuse. Sand y décrit le dilemme amoureux de l'héroïne en

15. Летопись жизни и творчества А. И. Герцена, 1812-1870, t. I : 1812-1850, Moskva, AN SSSR, 1974, p. 514.

16. Р. A. Annenkov, « Замечательное десятилетие », in Гериен в воспоминаниях современников, Moskva, 1956, p. 127-175, ici p. 170.

17. Lina Steiner, «Gercen's tragic Bildungsroman: love, autonomy, and maturity in Aleksandr Gercen's Byloe i dumy », Russian literature, t. LXI, 2007, fasc. I-II, p. 139-174.

18. XXII : 101. Les chiffres romains renvoient aux tomes de l'édition « académique » des CEuvres de Herzen [A. I. Gercen, Собрание сочинений в тридиати томах, Moskva, AN SSSR, 1954-1965], les chiffres arabes aux pages.

19. Kate Holland, "Literary contexts of triangular desire : Natal'ja and Aleksandr Gercen as readers of George Sand », Russian literature, t. LXI, 2007, fasc. I-II, p. 175-205. 
titre, Consuelo, ballottée en tous sens entre un mystérieux intrigant Liverani et son ancien amant Albert. Tiraillée entre les deux, elle finit par opter pour Albert, mais comprend un peu plus tard que Liverani et Albert sont une seule et même personne.

Dans un premier temps, autant Herzen qu'Herwegh pouvaient percevoir dans cette constellation deux aspects : d'une part une identité idéale-typique de leur point de vue et d'autre part une séduisante possibilité d'ouvrir leur propre mariage pour leur ami. Suite à la catastrophe, Herzen se référait sans cesse au roman de Sand Horace. Le protagoniste y apparaît comme un dandy qui joue toujours un rôle devant lui-même et les autres. C'est pourquoi il échoue autant politiquement que dans sa vie privée : il est trop fainéant pour guider une révolution, et n'ose pas non plus consacrer sa vie à sa bien-aimée.

Dans Passé et méditations, Herzen évoque ce roman comme occupant une place centrale pour lui. Lors de sa dernière rencontre avec Herwegh, il lui demande s'il a lu Horace ${ }^{20}$. Herwegh apparaît à Herzen comme une figure romanesque faible, pareille à Horace, qui, au travers de sa personnalité égoïste et narcissique, entrave la vie d'un authentique héros révolutionnaire (tel que Herzen le voit encore à ce moment-là).

Avant même que la description du drame familial proprement dit ne commence, Herwegh est déjà présent sur plus de pages dans les dernières parties du chapitre "la fièvre typhoïde", sans que Herzen ne le nomme explicitement. Dans cette introduction narrative retenue, Herzen assortit la forme obscure de Herwegh d'une aura de danger, qui, à travers la fraternisation apparente avec la bonne cause, apparaît particulièrement abominable.

Dans Passé et méditations, Herzen affirme en recourant à la personnalité d'Horace qu'Herwegh lui aurait été dès le début antipathique ${ }^{21}$ :

Il ne disposait pas de cette nature simple, sincère, de cet abandon complet de soi, qui va si bien à tous les êtres talentueux et les forts et qui est chez nous inséparable du fait d'être doué. Il était renfermé, rusé, il craignait les autres, et se divertissait volontiers en secret. Il était de nature capricieuse, objet d'une dépendance pitoyable aux bagatelles, au confort de la vie et était d'un égoïsme infini, sans égards, qui n'avait pour limite que la naïveté du cynisme ${ }^{22}$.

\footnotetext{
20. X : 260 .

21. Le jugement de Herzen coïncidait avec celui de Franz Liszt qui écrivait le 10 octobre 1843 : « Herwegh me paraît bien inconsistant - il faudrait à mon sens faire un article aux deux tiers critique pour être dans la moyenne vraie - un peu insister sur ce manque d'expérience de la vie et de l'histoire - sur la fausse poésie de l'amour partagé entre sa maîtresse et la liberté. » (Lettre de Franz Liszt à Marie d'Agoult, 10 oct. 1843, dans Correspondance de Franz Liszt avec Marie d'Agoult, éd. critique Serge Gut et Jacqueline Bellas, Paris, Fayard, 2001, p. 1027).

22. $\mathrm{X}: 240$.
} 
La disqualification morale de Herwegh que Herzen a, selon sa propre déclaration, effectuée au premier regard, s'opère en réalité bien plus tard. Dans des lettres à ses amis russes, Herzen appelle encore Herwegh à la fin des années 1840 « son unique camarade de mentalité en Occident ${ }^{23}$ ».

Cette date précédant l'aversion de Herzen pour Herwegh ne doit toutefois pas simplement démontrer l'infaillibilité de sa connaissance de l'homme, mais illustrer la figure de Herwegh à tous égards comme contrastant avec son caractère propre. Herzen parle explicitement de la "divergence » de leurs caractères ${ }^{24}$. Herzen lui-même est le «talentueux » et le « fort », duquel le " gâté » Herwegh se tient de manière ingrate en retrait. La preuve que Herzen cherche à fournir après coup, à la fois de l'existence et de la non-existence d'une fiabilité politique et privée apparaît plus importante encore que le contraste des caractères. L'attaque de Herzen contre Herwegh renferme aussi une dépréciation de sa production poétique. Herwegh n'aurait pas simplement cédé à un désir érotique mais se serait infiltré par faiblesse humaine dans le couple d'un ami. La tromperie privée est en même temps une trahison politique. Herwegh, l' « épicurien bourgeois », n'a pas le courage d'un combat ouvert - ni lors du conflit avec Herzen, ni lors du soulèvement badois qu'il a fui comme un lâche ${ }^{25}$. Lors de la description de l'année 1852, Herzen rend finalement son verdict définitif à propos de Herwegh : un petit bourgeois allemand, incapable d'actes et capable seulement de mots vides $^{26}$. Ce reproche est révélateur: en tant qu'allemand, Herwegh tire encore de la tragédie personnelle d'un autre un profit financier, en tant que petit bourgeois, il ne recherche que son propre bien-être ${ }^{27}$.

Herwegh recourt également à George Sand dans l'interprétation du drame amoureux, mais à un autre roman cette fois, à savoir la Petite Fadette (1848). Au centre de ce drame amoureux se trouve un couple de jumeaux, Landry et Sylvinet. Landry et Sylvinet ont été élevés différemment : Landry est viril et affirmé, tandis que Sylvinet arbore un côté féminin, laissant souvent libre cours à ses sensations. L'intrigue s'intensifie lorsque Landry entreprend une liaison avec Fadette. Sylvinet réagit avec jalousie à l'intrusion de cette jeune fille dans leur relation fraternelle symbiotique.

Dans des tentatives désespérées de réconciliation au début de l'année 1850, Herwegh se décrit sans cesse comme Sylvinet et interpelle Herzen en tant que « besson $^{28}$ ». Herzen est donc de cette manière poussé dans le rôle de Landry, personnage du roman de George Sand, et sert ainsi à Herwegh d'objet d'amour courtois. Herzen accepte dans l'immédiat cette interpré-

23. Lidija Ginzburg, Былое и думы Гериена, Moskva, 1957, p. 301.

24. $\mathrm{X}: 251$.

25. $X: 243-247$.

26. $\mathrm{X}: 285$.

27. $X: 266,251$.

28. Lettres $61,65,81 \mathrm{f} ., 84,88 \mathrm{f}$. 
tation littéraire. Après l'élimination d'un désaccord, il écrit à Herwegh le 22 janvier 1850 :

Je vous embrasse de tout mon cœur pour votre lettre, cher Georg; je l'ai lue et relue, oui, oui, nous restons, nous resterons ce que nous sommes... amis, jumeaux. Je vous le jure ! ${ }^{29}$

L'exaltation rhétorique laisse pourtant bientôt place à un désenchantement. Pas tant chez Herwegh qui se rabat sur des modèles littéraires toujours neufs, pouvant éclairer et légitimer sa relation. Dans une lettre à sa femme Emma, Herwegh actualise justement le dilemme des affinités électives. Bien qu'il ait violé l'institution de toute façon sans valeur du mariage, il n'a pourtant pas porté atteinte à l'amour éternel :

Je commence à y trouver du sens, bien sûr pas celui que la morale y met habituellement. Le mariage n'est pas sacré, mais l'amour l'est, et un amour dans le mariage est profané, entaché sans arrêt, parce que le mariage contraint l'amour à l'hypocrisie, au faux-semblant, à un rôle qui est indigne de lui ${ }^{30}$.

L'argumentation d'Herwegh aboutit à ce que sa trahison amoureuse devienne finalement une sincérité. Si en effet sa passion pour Natalie n'avait pas fléchi, il aurait alors souillé son amour sacré pour Emma en jouant la comédie.

Le 7 juillet 1850 Herwegh rapporte à Herzen qu'il lit la nouvelle la Pie voleuse (Sorovka-vorovka) ${ }^{31}$. Dans ce mélodrame, les catégories d'héroösme et de lâcheté sont mélangées - un héros de guerre et un déserteur sont confondus, un reproche de vol se révèle erroné. Avec la mention de cette lecture, Herwegh signale qu'il est accusé à tort.

Le modèle littéraire ultime pour sa propre passion est en fait le Nouveau Testament. Herwegh parle de sa "crucifixion», et ne sait pas s'il pourra vivre sa «résurrection ${ }^{32}$. L'identification avec le fils de Dieu apparaît même explicitement dans une lettre, lorsque Herwegh appelle à lui les enfants de Herzen : «Laissez les enfants venir à moi - j'ai une grande envie de dire comme Jésus Christ - Vivent les petits ${ }^{33}$.»

En janvier 1851, Herwegh écrit une lettre pathétique à Herzen, dans laquelle il le conjure une dernière fois de comprendre la nécessité de son amour pour Natalie :

Le caractère irrésistible d'une attraction ancrée dans l'essence et la quintessence de nos deux natures, celle de Natalie et la mienne, la néces-

29. L. P. Lanskij, « Письма [Герцена] к Георгу и Эмме Гервегам », in Литературное наследство, t. 64,1958 , p. 9-318, iсi p. 81.

30. Lettre 71.

31. Lettre 92.

32. Lettre 65.

33. Lettre 87. 
sité qui lie nos deux âmes ensemble; cela, Alexandre, je n'avais ni le droit ni le pouvoir de le fuir! Et en effet qui irait jusqu'à se fuir luimême? J'aurais fui, je l'aurais fait si un quelque acte de ma part avait soulevé ou provoqué ce sentiment en elle, s'il n'y avait pas eu de réciprocité, de simultanéité apparue comme une sorte d'éclair... L'amour était là, plus fort que nous ${ }^{34}$.

L'insistance sur la nécessité fatale et naturellement élémentaire de leur affaire amoureuse apparaît ici comme fondamentale, et cet argument romantique sonne également comme familier à Herzen.

Finalement, entre Herzen et Herwegh, ce sont deux conceptions romantiques différentes qui s'affrontent. Les deux auteurs alignent explicitement leur relation sur des modèles littéraires et identifient aussi les partenaires du drame avec des figures appartenant à ces intrigues.

Herzen décrit Herwegh dans Passé et méditations comme le nouveau "Werther ${ }^{35}$ » et le caractérise comme un héros byronien :

Herwegh a d'une certaine manière concentré la lumière sur lui ; il s'est abandonné à la cupidité, a cherché l'attention ; timidement mais plein d'amour-propre il était en même temps sûr de lui et certain de sa supériorité. Tous ces éléments réunis l'ont poussé à des coquetteries, des caprices, à être parfois à dessein triste, attentif ou inattentif. Il avait constamment besoin d'être l'élément dirigeant, le favori. À la fois ami et esclave (précisément comme Emma) pouvant supporter le froid et les reproches, même lorsque son service n'était pas nécessaire, et, qui, à première vue, était prêt de nouveau à s'élancer à corps perdu et exécuter avec un sourire et obéissance ce qu'on lui demandait ${ }^{36}$.

Au point culminant de la querelle, Herwegh renverse les rôles et accuse Herzen de rationalisme exagéré. Le 4 janvier 1850, il écrit :

Le diable de l'analyse vous pousse à vouloir toujours arriver trop vite à une formule; amitié et amour doivent passer dans votre creuset $\operatorname{logique}^{37}$.

Et un peu plus tard il lui reproche de se perdre dans des chaînes de déduction logiques :

D'une seule histoire, d'un seul caprice vous tirez des conclusions à l'infini. Est-ce que le diable nous pousserait à nous perdre l'un l'autre? ? $^{38}$

Herwegh le formule plus clairement dans une lettre du 26 janvier 1850, dans laquelle il reproche à Herzen un usage global du concept romantique,

34. Carr, op. cit., p. 66. Cette lettre ne se trouve que là. Pour l'histoire de sa publication, voir Lettre 528.

35. $\mathrm{X}: 257$.

36. $\mathrm{X}: 251$.

37. Lettre 25.

38. Lettre 29. 
sous lequel celui-ci comprend tout ce qui n'est pas explicable rationnellement :

Elle est restée aussi réelle qu'elle l'a été, et c'est à tort que vous me reprochez de l'avoir entraînée dans un certain romantisme.

Est-ce que vous finirez par appeler romantique toute manière de voir le monde autrement que par les lunettes de la simple compréhension ? ${ }^{39}$

Déjà avant ce conflit ce reproche était apparu dans la correspondance du couple d'Herwegh. Le 2 août 1849, Herwegh écrit à Emma :

Ni Natalie ni Herzen ne développent le moindre talent pour s'accommoder de la vie, si l'on peut appeler cela une vie, et ne sont pas capables, malgré toute leurs grandes théories, ne serait-ce que de profiter d'une heure de liberté réelle ${ }^{40}$.

À côté du reproche de rationalisme, Herwegh critique aussi l'inconséquence entre les théories de Herzen et leur mise en pratique. Herzen voudrait donner la liberté à la femme, mais il se comporte avec sa propre épouse comme un despote :

J'attends Herzen à l'épreuve pratique de ses théories. Apparemment il donne toute la liberté à sa femme - mais il dit cela d'une manière qui ressemble beaucoup à une contrainte morale. Qu'il ait de l'affection pour elle, je n'en doute pas, mon Dieu, il est venu des frontières de la Sibérie pour l'enlever, mais que la passion est passée je le sais aussi bien -- que la femme a beaucoup d'affection pour lui, mais qu'il ne peut plus la satisfaire, elle lui l'a dit franchement, en lui déclarant qu'elle m'aime, $\mathrm{moi}^{41}$.

Avec ce reproche de rationalisme, Herwegh manque pourtant sa cible. Plus encore : après la rupture avec Herzen, il s'autorise même - malgré son «cœur plus chaud»- un «jugement plus froid ${ }^{42}$. Mais Herzen n'est en aucune façon le rationaliste froid qu'Herwegh voulait voir. Le projet de vie global de Herzen était porté par un missionariat romantique. Il croyait à son élection et se considérait franchement comme le fondateur d'une nouvelle Russie $^{43}$. Son programme de rédemption n'était justement pas dû à un calcul rationnel, mais à un enthousiasme romantique.

Ce dévouement romantique est utilisé également dans une ébauche de l'Autobiographie de Herzen, afin justement de légitimer pour lui-même ce crime, qu'il ne pouvait pardonner à Herwegh, celui de l'adultère. En 1843, il

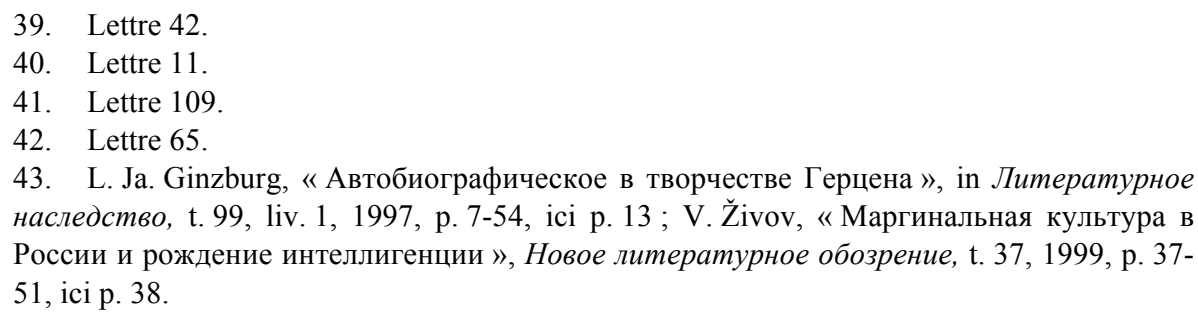


commet une infidélité avec une femme de chambre. Pour des raisons évidentes, Herzen n'a pas conservé ce passage dans la version définitive de Passé et méditations :

Lorsqu'un jour je rentrai tard à la maison, je dus rentrer par la porte de l'arrière de la maison. Katerina m'ouvrit la porte. On voyait qu'elle venait à peine de quitter son lit, ses joues étaient encore rosies par le sommeil, elle portait un châle renversé, et sa natte épaisse relevée était prête à s'effondrer à la manière d'une lourde vague... C'était le petit matin. Elle me regarda et dit en souriant : « Comme vous arrivez tard. »

Je la regardai, enivré par sa beauté, et posai instinctivement, à demiconscient, ma main sur son épaule, le châle tomba... Elle poussa un cri... Sa poitrine était découverte.

Que faites-vous, chuchota-t-elle, et me fixa d'un regard plein de trouble puis se détourna, comme pour me laisser sans témoins... Ma main toucha son corps brûlant de sommeil... Comme la nature est belle lorsque l'homme s'abandonne à elle en s'oubliant lui-même, en se perdant en elle...

À cet instant-là j'aimai cette femme, et il y avait quelque chose d'immoral dans cet enivrement... Quelqu'un avait été blessé, rabaissé... Qui ? L'être qui m'était le plus proche et le plus cher au monde. Mon emballement passionné avait un caractère trop éphémère pour s'emparer de moi - il n'avait aucune racine (ni d'un côté ni de l'autre, et de sa part à elle il n'y avait sans doute pas d'emballement), et tout se serait déroulé sans laisser de trace, en ne laissant qu'un sourire, un souvenir fiévreux et peut-être deux fois l'épanouissement d'une rougeur sur les joues...

Il n'en fut pas ainsi, d'autres forces intervinrent. J'avais sans réfléchir fait rouler une pierre... Il n'était plus en mon pouvoir de l'arrêter ou de la maitriser ${ }^{44}$.

Herzen justifie cette affaire en désignant deux instances romantiques : l'amour et la nature. Il n'entend ici pourtant pas l'amour envers la femme de chambre mais son amour pour Natalie, qui apparaît comme la femme idéale derrière le partenaire sexuel, les deux se fondant en une personnification de l'éternel féminin. La nature est aussi présente dans la métaphore de la pierre qui roule, et du point de vue de Herzen ce n'est pas seulement insensé, mais même immoral de s'opposer aux forces de la nature.

De la même manière qu'Herwegh, Herzen fait entrer en ligne de compte un argumentaire hasardeux. Le 24 mars 1851, Herwegh justifie son infidélité comme un phénomène naturel et rejette le reproche de trahison :

Et puis - elle est ma femme devant - j'ai voulu dire Dieu - pardon ! Et devant la nature - je n'ai rien à conquérir [...]. Il ne s'agit pas de trahison ${ }^{45}$.

44. IX : 97.

45. Lettre 114. 
Cette justification aventureuse confirme la fantaisie narcissique des deux hommes adultères, puisqu'on peut couronner l'amour divin avec en plus la bénédiction du mari cocu.

Tout ce qui se passe entre moi et Natalie est sanctifié par un abandon complet de l'un à l'autre, une perte de l'âme dans celle de l'autre, jusqu'à un point où nous pouvions nous rencontrer dans des régions vierges de toute respiration humaine, dans des régions où l'individu se tient tremblant devant lui-même, où il ne se confessera pas, même à soi, mais où il peut être divinisé par un autre, par un seul être dans l'univers, et c'est là que j'ai rencontré Natalie. Combien de fois nous nous sommes exclamés : Si Alexandre avait compris ! S'il avait pu comprendre ! Il serait tombé à genoux et aurait adoré ! ${ }^{46}$

Cette réconciliation imaginaire apparaît encore dans une lettre à François Wille écrite à l'occasion de la mort de Natalie :

Natalie est morte. Il ne s'agit que d'une mort extérieure, séparée de l'intérieur, et que le monde appelle le déclin, la maladie, la pleurésie, la fausse couche. Je savais tout, je savais que Herzen l'avait assassinée, d'où ma folie de vouloir le tuer. Ce sentiment s'atténue à présent, et je ne crois pas que nous nous étranglerons sur cette tombe. Les apparences sont sauves et il ne sait pas que la vérité l'a fait périr. Mais il est misérable et seul comme moi. Si nous étions des hommes, Herzen et moi, nous devrions nous tendre la main. En sommes-nous ? $?^{47}$

Au milieu du mois de janvier 1852, Herwegh affirme contre Herzen, qu'il s'agissait seulement pour lui et Natalie du rétablissement de l'unité de l'amour et de l'amitié dans le carré formé par Herzen et Herwegh. Le maximalisme de cette revendication soulignait par-là que Herwegh supposait que son histoire avec Natalie ne pouvait constituer un état permanent, mais seulement une transition vers un ménage à quatre. Lorsque cette présentation idéale fut défaite, seul le suicide collectif à la Heinrich von Kleist apparaissait possible :

Vous savez enfin que l'idéal de notre vie pour N. et moi était la réparation du malheureux accident de G., qu'elle ne rêvait et ne pensait qu'à un enfant de moi, que là était tout notre avenir qu'elle croyait y être arrivé lorsqu'elle vous a parlé - - - peut-être vous ne savez pas, que je ne suis resté jusqu'à la catastrophe que parce que N. m'avait juré de « vouloir s'arracher à tout », nous tuer, le pistolet ${ }^{48}$.

46. Carr, op. cit, p. 66.

47. Ingrid Pepperle, «Georg Herwegh und Alexander Herzen: Unbekannte Briefe zum Bruch ihrer Beziehungen von Richard Wagner, François Wille und Georg Herwegh ", in Begegnung und Zeiten: Festschrift für Helmut Richter zum 65. Geburtstag, éd. Regina Fasold, Christine Giel, Volker Giel, Michael Masanetz, Michael Thormann, Leipzig, 1999, p. 141-153.

48. Lettre 144. 
Herzen n'accepte bien entendu pas cette représentation, c'est pourquoi Herwegh passe de la défense à l'attaque. Le 16 février 1852, il écrit à Emma :

Le pacte est conclu et valable éternellement. Mon âme appartient à cet amour ou au diable. [...] Sa vie avec lui [Herzen] ne serait-elle pas une fornication continue ? $^{49}$

Herwegh renvoie alors rapidement la balle: Natalie était sa femme « naturelle », alors que la vie en commun dans le mariage avec Herzen apparaissait comme une «fornication». Herwegh joue ici l'amour-nature romantique contre le corset contraignant du mariage, dans lequel il ne pouvait par définition pas y avoir d'amour.

Le drame familial de Herzen était alors narré sous deux facettes dans différentes intrigues romantiques : une trahison noire, un amour fatal, la fusion de l'amitié et de l'érotisme, le mariage despotique et finalement l'union de l'amour et de la mort.

Herzen expose sa version littéraire du drame familial à tous les esprits dirigeants de l'Europe, Proudhon et Michelet en reçoivent des lettres détaillées, George Sand est informée par son amant Müller-Strübing, et même Karl Marx sera au courant de cette histoire ${ }^{50}$.

Herwegh conservera aussi jusqu'à un âge avancé la mise en forme littéraire de celle-ci. Malwida von Meysenbug, la gouvernante et plus tard la mère adoptive d'Olga, la fille de Herzen, s'adressa à Herwegh en 1872 à l'instigation de Richard Wagner avec le souhait que lui soient rendues les lettres d'amour de Natalie. Herwegh réagit avec un rejet abrupt : « Ni maintenant, ni plus tard, ni jamais. » Cette « affaire» concerne quatre personnes seulement, et «sans ce crime» personne n'aurait jamais pu être mis au courant du secret. Il ne peut pas croire que, «pour le couronnement de l'édifice », l'on exige que le héros du roman livre les lettres de l'héroïne ${ }^{51}$.

En fin de compte, Herwegh réclame pour lui le monopole de l'interprétation de l'affaire, ne voulant passer ni pour un homme adultère ni pour un traître; c'est pourquoi il imagine son amour envers Natalie dans une relation romantico-utopique à quatre, qui doit rester secrète. 